\title{
Complementary use of aesculin hydrolysis and inositol fermentation in the characterisation of Klebsielleae
}

\author{
J. G. BARR AND REBECCA J. MAHOOD
}

From the Department of Clinical Bacteriology, Royal Victoria Hospital, Belfast, Northern Ireland

SUMMARY Rates of aesculin hydrolysis and inositol fermentation adequately differentiate Klebsielleae from other Enterobacteriaceae. In combination with tests for motility and growth in potassium cyanide medium, presumptive differentiation between Klebsielleae, and confirmation of Klebsiella pneumoniae, is obtained.

Aesculin hydrolysis and inositol fermentation have frequently been incorporated with success in biochemical testing schemes for differentiation between clinical isolates of Enterobacteriaceae.

Wasilauskus (1971) demonstrated that Klebsielleae could be readily differentiated from other Enterobacteriaceae by virtue of hydrolysis of aesculin to 6,7 dihydroxy coumarin, the reaction product combining with iron to form a black compound.

Inositol fermentation has also been widely used in differentiation of the Enterobacteriaceae, and inositol has been incorporated in specific screening media (Donovan, 1966; Barr and Mahood, 1976) and primary isolation medium (Davis and Matsen, 1974) for Klebsielleae. Barr and Mahood (1976), however, showed that some clinical isolates of Klebsiella spp, particularly Klebsiella pneumoniae and $K$. ozaenae, fermented inositol weakly and slowly, and demonstrated that fermentation by other Klebsiella spp could also appear to be weak or absent due to a rapid conversion of acid to neutral metabolites.

Since both aesculin hydrolysis and inositol fermentation have been utilised in parallel in some schemes for differentiation of Enterobacteriaceae (Chadwick et al., 1974), this investigation was carried out to elucidate and compare the ability of Klebsielleae and other Enterobacteriaceae to metabolise these two substrates. The results obtained were utilised to assess the value of the complementary use of aesculin hydrolysis and inositol fermentation, in an inositol-hydrogen sulphide-motility medium (Barr

Received for publication 24 May 1976 and Mahood, 1976), in the identification of Klebsielleae.

The value of these observations in the identification of Enterobacteriaceae by a replica agar plating method, modified from that described by Chadwick et al. (1974), is discussed.

\section{Material and methods}

\section{ORGANISMS}

Clinical isolates A total of 710 clinical isolates of Gram-negative bacilli examined comprised 434 cultures derived from urinary tract infections and $\mathbf{2 7 5}$ cultures isolated in the routine bacteriological laboratory from other sources. Of these, 234 cultures were identified as Klebsielleae by the methods of Chadwick et al. (1974) and Cowan (1974). An additional 20 clinical isolates of Klebsiella spp were obtained from Ruchill Hospital, Glasgow.

Stock cultures The following 26 cultures were also examined: Klebsiella aerogenes NCTC 8172; $K$. atlantae NCTC 9496; $K$. edwardsii NCTC 5054; $K$. pneumoniae (4) (NCTC 9633, 204, 8632, and 10246); K. ozeanae (4) (NCTC 5050, 9659, 10313, and 8883); $K$. rhinoscleromatis (2) (NCTC 5046 and 5047); Enterobacter aerogenes NCTC 10006; Enterobacter cloacae NCTC 10005; Serratia marcescens NCTC 9940; Escherichia coli NCTC 10418; Proteus mirabilis NCTC $5887 ; P$. morganii NCTC $235 ; P$. vulgaris NCTC 4175; $P$. rettgeri NCTC 7475; Providencia B, subgroup B, NCTC 10318; Providencia spp NCTC 10286; Citrobacter freundii NCTC 
9750; Pseudomonas aeruginosa NCTC 10332; and Ps. aeruginosa NCTC 10701.

SCREENING MEDIA PREPARATION

Seven basic screening media were employed. Aesculin bile agar (Difco), Simmon's citrate agar (Difco), Phenylalanine agar (Difco), Xylose-lysinedeoxycholate agar (Difco), and Ornithine decarboxylase agar, derived from the fluid decarboxylase medium of Fay and Barry (1972), were utilised as described by Chadwick et al. (1974). Two further screening media employed were inositol-hydrogen sulphide-motility agar medium (Barr and Mahood, 1976) and a potassium cyanide (KCN) agar medium based on that described by Braun (1938).

The inositol-hydrogen sulphide-motility medium contained (g/l): Tryptone (Oxoid), 10.0; $\mathrm{NaCl}$, 5.0; Agar (Oxoid), 3.0; 2,3,5, triphenyl-tetrazolium chloride, $0.05 ; \mathrm{Fe}_{2} \mathrm{NH}_{4} \mathrm{PO}_{4}, 0.3 ; \mathrm{Na}_{2} \mathrm{~S}_{2} \mathrm{O}_{3} .5 \mathrm{H}_{2} \mathrm{O}$, $0.2 ; \mathrm{K}_{2} \mathrm{HPO}_{4}, 0.3$; acetoin, 8.5; and inositol, 20.0. Bromothymol blue in aqueous solution was added to give a final concentration of $0.003 \%$. The medium was sterilised by autoclaving at $115^{\circ} \mathrm{C}$ for 10 minutes, acetoin and 2,3,5, triphenyltetrazolium chloride were added as filter sterilised aqueous solutions, and the $\mathrm{pH}$ value was adjusted to $6 \cdot 6$.

Potassium cyanide agar medium was prepared from KCN broth medium (Braun, 1938; Møller, 1954), described by Cowan (1974), and supplemented with $0.5 \%$ agar (Oxoid). Potassium cyanide was added from a $0.5 \%$ aqueous solution to give a final concentration in agar of $0.125 \mathrm{~g} / 1$ (1 part in 8000). Agar was cooled to $45^{\circ} \mathrm{C}$ before addition of $\mathrm{KCN}$, dispensed immediately, and allowed to solidify at $4^{\circ} \mathrm{C}$. Medium was freshly prepared and distributed on each day of use, and sensitivity was checked at frequent intervals with appropriate stock cultures.

Sterilin replidishes were used throughout, $3 \mathrm{ml}$ of each medium except inositol-hydrogen sulphidemotility medium $(4 \mathrm{ml})$ being dispensed in each compartment.

\section{INOCULATION AND INCUBATION}

All media were inoculated from a four-hour peptone water culture. In specific investigations sequential 10-fold dilutions of inocula were prepared in $9 \mathrm{ml}$ peptone water. Motility medium was inoculated with a straight wire and other media with one drop from a Pasteur pipette. The peptone water culture used for inoculation of other media was reincubated for a further 16 hours at $37^{\circ} \mathrm{C}$.

Ornithine decarboxylase agar was covered with liquid paraffin before incubation and all plates were incubated for 16 hours at $37^{\circ} \mathrm{C}$.
INTERPRETATION OF BIOCHEMICAL REACTIONS

Utilisation of inositol, aesculin, and ornithine $\mathrm{was}^{+}$ detected by medium colour change, and citrate utilisation by a colour change and/or visible growth Resistance to $\mathrm{KCN}$ was recorded by growth, anf colony colour on xylose-lysine-deoxycholate agap was noted. Production of indole in peptone wate was tested with Kovac's reagent, and phenyl $\overrightarrow{0}$ alanine deaminase activity was detected by the addition of acidified $10 \%$ ferric chloride solution $\vec{\omega}$ Hydrogen sulphide production and motility were recorded after 16 hours, and again after a further 245 hour incubation period.

A semiquantitative determination of aesculiñ hydrolysis and inositol fermentation was recorded Aesculin hydrolysis was recorded after 4 hours an $\phi$ 16 hours as - (no hydrolysis), ++ (distinct colour change usually limited to inoculation surface), and +++ (complete colour change within an individuaz inoculation compartment). Inositol fermentation waఖ recorded as - (no fermentation), + (indicator change along line of stab inoculation), ++ (cleap zone of indicator colour change), and +++ (com-v plete indicator change within individual inoculaø tion compartment).

IDENTIFICATION OF CLINICAL ISOLATES

Clinical isolates were identified as far as possible using the basic biochemical differentiation scheme described by Chadwick et al. (1974). Where furthe 5 tests were necessary these were carried out by con ventional methods (Cowan, 1974).

Identification of Klebsiella isolates was confirmed: by further biochemical tests by conventionas methods, viz, methyl red and Voges-Proskauero fermentation of dulcitol, inositol, and glucose (gas) malonate and gluconate utilisation; and urease् activity.

\section{Results and discussion}

The hydrolysis of aesculin and fermentation of inositol by 730 clinical isolates and 26 stock cultures of Gram-negative bacilli are summarised in Table 10 National Collection of Type Culture strains ex amined gave reactions typical of the majority $Q \Phi$ clinical isolates of the same species.

\section{HYDROLYSIS OF AESCULIN}

Among the Gram-negative bacilli investigated, the genera Klebsiella, Enterobacter, and Serratia were readily distinguished from other genera by rapi® hydrolysis of aesculin. Sixty-three per cent of Klebsielleae showed visible hydrolysis, by formao tion of the black reaction product after four hours 
Table 1 Hydrolysis of aesculin and fermentation of inositol by 756 isolates of Gram-negative bacilli

\begin{tabular}{|c|c|c|c|c|c|c|c|c|c|c|c|}
\hline & \multirow{3}{*}{$\begin{array}{l}\text { Total } \\
\text { number } \\
\text { of } \\
\text { isolates }\end{array}$} & \multicolumn{6}{|c|}{ Hydrolysis of aesculin 1} & \multicolumn{4}{|c|}{ Fermentation of inositol ${ }^{2}$} \\
\hline & & \multicolumn{3}{|c|}{4 Hours } & \multicolumn{3}{|c|}{16 Hours } & \multicolumn{4}{|c|}{16 Hours } \\
\hline & & - & ++ & $+t+$ & - & ++ & $++t$ & - & + & ++ & $+t+$ \\
\hline \multicolumn{12}{|l|}{ Klebsiella aerogenes/oxytocal } \\
\hline$K$. pneumoniae & $\begin{array}{r}202 \\
15\end{array}$ & 11 & $\begin{array}{r}142 \\
4\end{array}$ & $\begin{array}{l}0 \\
0\end{array}$ & $\mathbf{0}$ & 0 & $\begin{array}{r}202 \\
15\end{array}$ & $\begin{array}{l}1 \\
6\end{array}$ & 8 & $\begin{array}{r}10 \\
1\end{array}$ & $\begin{array}{r}170 \\
0\end{array}$ \\
\hline K. ozaenae & 12 & 7 & 5 & 0 & 0 & 1 & 11 & 2 & 4 & 6 & $\mathbf{0}$ \\
\hline$K$. rhinoscleromatis & 4 & 0 & 4 & 0 & 0 & 0 & 4 & $\mathbf{0}$ & 2 & 2 & $\mathbf{0}$ \\
\hline Enterobacter aerogenes & 16 & 10 & 6 & $\mathbf{0}$ & 0 & 3 & 13 & 0 & 2 & 1 & 13 \\
\hline Enterobacter/Serratia ${ }^{3}$ & 33 & 20 & 13 & $\mathbf{0}$ & $\mathbf{0}$ & 4 & 29 & 17 & 8 & 7 & 1 \\
\hline Proteus mirabilis & 56 & 56 & 0 & 0 & 56 & 0 & 0 & 56 & 0 & 0 & $\mathbf{0}$ \\
\hline P. vulgaris & 5 & 5 & 0 & 0 & 2 & 3 & 0 & 5 & 0 & 0 & 0 \\
\hline P. morganii & 11 & 11 & 0 & 0 & 10 & 1 & 0 & 8 & 3 & $\mathbf{0}$ & 0 \\
\hline$P$. rettgeri & 20 & 20 & 0 & $\mathbf{0}$ & 15 & 3 & 2 & 4 & 2 & 3 & 11 \\
\hline Escherichia coli & 316 & 316 & 0 & 0 & 302 & 10 & 4 & 300 & 15 & 1 & 0 \\
\hline Citrobacter spp. & 44 & 44 & 0 & 0 & 41 & 3 & 0 & 41 & 2 & 1 & 0 \\
\hline Providencia spp. & 3 & 3 & 0 & 0 & 3 & $\mathbf{0}$ & 0 & 2 & 0 & 1 & 0 \\
\hline Pseudomonas aeruginosa & 22 & 22 & 0 & $\mathbf{0}$ & 19 & 3 & 0 & 22 & 0 & 0 & 0 \\
\hline
\end{tabular}

${ }^{1}$ Hydrolysis of aesculin was recorded as $(-)$ no hydrolysis, $(++)$ clear zone of colour change due to hydrolysis, $(+++)$ complete change within inoculation compartment.

${ }^{2}$ Fermentation of inositol was recorded as $(-)$ no fermentation, $(+)$ indicator change along line of stab inoculation, $(++)$ clear zone of indicator colour change, $(+++)$ complete indicator change within individual inoculation compartment.

${ }^{3}$ Isolates include Enterobacter spp. and Serratia spp. other than Enterobacter aerogenes.

NB Apart from Klebsielleae only five isolates, all Proteus rettgeri, gave a positive reaction on both substrates.

incubation, and aesculin hydrolysis of all Klebsielleae, excepting one, resulted in a complete colour change within the inoculation compartment after 16 hours' incubation.

No isolates of non-Klebsielleae exhibited any hydrolysis of the substrate after four hours' incubation. Although $27(5 \cdot 6 \%)$ of non-Klebsielleae isolates screened showed detectable hydrolysis of aesculin after 16 hours, hydrolysis by only six isolates resulted in a complete colour change within the inoculation compartment. Indeed, with the exception of four isolates of $E$. coli and two isolates of $\boldsymbol{P}$. rettgeri, aesculin hydrolysis by non-Klebsielleae was restricted to the vicinity of bacterial growth on the surface of inoculation.

Inoculation with dilutions, prepared from the original peptone water, demonstrated that reduction of inocula of Klebsielleae, from $10^{7}$ viable organisms per $\mathrm{ml}$ to $10^{2}$ viable organisms per $\mathrm{ml}$, did not result in diminution in aesculin hydrolysis within 16hour incubations. Similar dilutions of inocula of non-Klebsielleae resulted only in a change in aesculin hydrolysis of Pseudomonas spp where a distinct reduction in activity was noted.

FERMENTATION OF INOSITOL

A semiquantitative determination of inositol fermentation by Gram-negative bacilli in an inositolhydrogen sulphide-motility medium was undertaken. As previously described (Barr and Mahood, 1976), and as indicated in Table 1, Klebsielleae could be divided into two groups on the basis of rate of inositol fermentation. K. aerogenes/oxytoca/atlantae/ edwardsii and Enterobacter aerogenes were normally rapid fermenters while $K$. pneumoniae, $K$. ozaenae, $K$. rhinoscleromatis, and Enterobacter/Serratia, other than Enterobacter aerogenes, were frequently slow or non-fermenters of inositol (Table 1).

Among non-Klebsielleae, 39 isolates (8.1\%) fermented inositol under the experimental conditions described. Eleven of 20 isolates of $P$. rettger $i$ gave a strong fermentation reaction comparable to that shown by the majority of the $K$. aerogenes/ oxytoca/atlantae/edwardsii and Enterobacter aerogenes group. Inositol fermentation by other nonKlebsielleae, including 15 isolates of $E$. coli, was recorded as weak.

COMPLEMENTARY VALUE OF AESCULIN HYDROLYSIS AND INOSITOL-HYDROGEN SULPHIDE-MOTILITY MEDIA

It has been shown here (Table 1) that during routine screening of Gram-negative bacilli, inositol fermentation by a considerable number of Klebsielleae was weak or absent. Difficulties in differentiation of Klebsielleae on this basis from other Enterobacteriaceae was compounded by the ability of many strains of $\boldsymbol{P}$. rettgeri to ferment inositol strongly and the demonstration of weak inositol fermentation, particularly by other Proteus isolates and by $E$. coli.

- The evidence presented demonstrates that Klebsiella spp and Enterobacter aerogenes could not be distinguished as a group on the basis of a semiquantitation of inositol fermentation alone.

However, all strains of Klebsielleae examined, excepting one, showed a similar rapid hydrolysis of 
Table 2 Typical test reaction patterns, given by rapid aesculin hydrolysing $(+++)$ isolates, on inositol-hydrogen sulphide-motility and potassium cyanide medium: percentage isolates of each species giving particular reactions are quoted

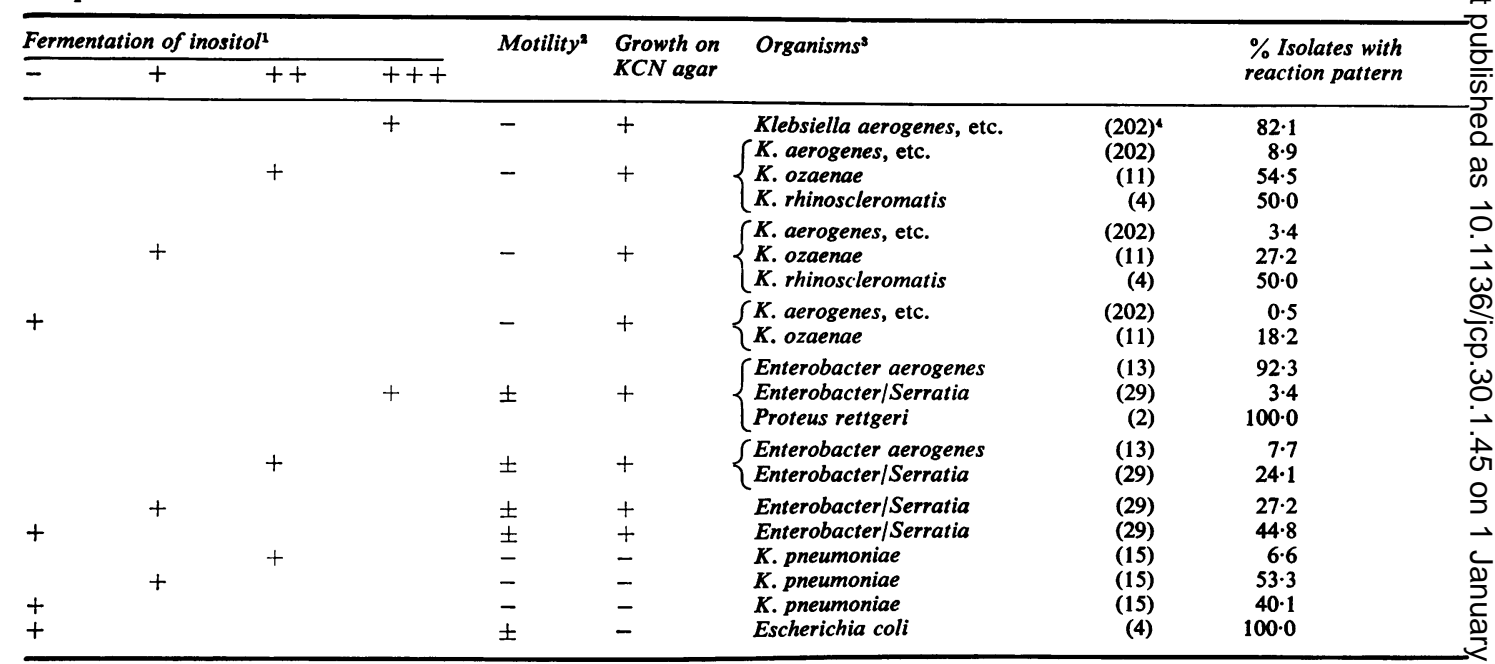

${ }^{1}$ Fermentation of inositol was recorded as $(-)$ no fermentation, $(+)$ indicator colour change along line of stab inoculation, $(++)$ clear zone of indicator colour change, $(+++)$ complete indicator change within individual inoculation compartment.

'Motility was recorded after 16 hours and after $\mathbf{4 0}$ hours' incubation. Motility recorded as ( \pm ) indicates motility in majority of isolates.

Total number of isolates of each species examined (bracketed).

'Klebsiella aerogenes, etc. includes $K$. aerogenes/oxytoca/atlantae/edwardsii.

aesculin which readily differentiated them as a group from other Enterobacteriaceae. Only six of 477 other isolates showed a comparable activity.

The results suggested that the use of these two media in parallel could be utilised in the differentiation of Klebsielleae and in the presumptive identification of particular species within that group. Among non-Klebsielleae investigated, only five isolates, all $P$. rettgeri, gave a positive reaction in both aesculin and inositol media.

An analysis of the results obtained for aesculin hydrolysis, inositol fermentation, and motility is summarised in Table 2. Growth in $\mathrm{KCN}$ medium was subsequently added to this biochemical test sequence to enable the differentiation of $K$. pneumoniae (Friedlander's bacillus) from other Klebsiella spp.

Since all excepting one Klebsielleae isolate investigated demonstrated evidence of rapid aesculin hydrolysis during 16-hour incubations, only strains showing strong aesculin hydrolysis have been included in Table 2 . No isolate giving rapid aesculin hydrolysis was shown to produce hydrogen sulphide in the inositol-hydrogen sulphide-motility medium. The results clearly demonstrate that the complementary use of these two test media would be of value in the presumptive identification and differentiation of Klebsielleae.

With the use of aesculin alone, six isolates only were encountered which gave rapid hydrolysis typical of Klebsielleae. With the use of an inositob screening medium alone a much larger number of non-Klebsielleae (39) was encountered, the majorits being differentiated from Klebsiella spp on the basis of motility. Only two isolates of $P$. morganii could be differentiated from motile Klebsielleae on the basiş of hydrogen sulphide production, and no inositol fermenting isolate of $P$. rettgeri, 16 in number produced hydrogen sulphide in the inositol-hydroger? sulphide-motility medium.

The use of the inositol medium in parallel $\operatorname{did}_{-}^{-}$ however, allow a presumptive differentiation among the Klebsielleae to be made. The non-motile rapid inositol-fermenting species $K$. aerogenes/oxytocas atlantae/edwardsii are differentiated particularly from $K$. pneumoniae and $K$.ozaenae, and among the motile Klebsielleae, Enterobacter aerogenes is differentiated from other Enterobacter spp and Serratia spp.

The utilisation of these two test media, and KCNP agar medium, in parallel may enable the diagnostiê bacteriologist to detect significant differences among isolates of Klebsielleae before embarking on extenfo sive biochemical testing.

VALUE OF AESCULIN AND INOSITOL-MOTILITY MEDIA IN BIOCHEMICAL TESTING SCHEMES FOR DIFFERENTIATION OF

ENTEROBACTERIACEAE

In this laboratory a modification of the scheme o⿺ Chadwick et al. (1974) has been adopted witlo 
success, particularly in the identification of Gramnegative bacterial urinary tract infections. The scheme incorporates indole production, phenylalanine deaminase activity, ornithine decarboxylase activity, aesculin hydrolysis, inositol fermentation, and growth on xylose-lysine-deoxycholate agar. Within this scheme, however, inositol has been maintained in a motility agar medium, and a $\mathrm{KCN}$ agar medium has been introduced. The latter medium enabled differentiation of $K$. pneumoniae from other Klebsielleae and contributed to the differentiation between other genera.

In this scheme the evaluation of ornithine decarboxylase activity enables the differentiation of Klebsiella spp from other Klebsielleae and overcomes the need for dependence on demonstration of organism motility. This is clearly an advantage, because of the intrinsic difficulty in predictably demonstrating motility in agar in some 16-hour incubations and in view of the prevalence among hospital Enterobacteriaceae of non-motile isolates of Serratia spp and Enterobacter spp (Oberhofer and Hajkowski, 1970).

By adopting this scheme, $E$. coli and $P$. rettgeri, which in a simple aesculin-inositol-motility screening regimen could be mistaken for Klebsielleae, are adequately differentiated. P. rettgeri is differentiated on the basis of phenylalanine deaminase activity. Rapid aesculin hydrolysing isolates of $E$. coli which, in the simple scheme, could not be differentiated from $K$. pneumoniae, except in the presence of an adequate demonstration of motility, are clearly separated on the basis of indole production. As far as we are aware, no reports of indole-positive $K$. pneumoniae have been noted, although indolepositive isolates of other Klebsielleae are frequently encountered (Rosner, 1970; Davis and Matsen, 1974). The rapid recognition of $K$. pneumoniae may be of special value, particularly if the widely held view that this species is potentially the most pathogenic Klebsiella spp was upheld.

In this laboratory, a compromise has been reached between the use of the simple biochemical testing scheme described and the more extensive biochemical identification screen. When staff are available during weekdays the complete range of tests are carried out and isolates are reported at genus or species level at the same time as antibiotic sensitivity reports are made.

At weekends, or other periods of staff shortage, only aesculin and inositol media are utilised. Although the limitations of this practice are noted, the system allows, in the majority of cases, the accurate reporting of isolates as Klebsiella spp, Proteus spp, or coliform species. For epidemiological studies, isolates examined and reported by the limited range of tests can be stored and identified at a later date.

We are grateful to Mrs W. Booth for preparation of this text and to Mr W. Dillon, Mr H. Walker, and Mr K. Curry for valuable assistance.

\section{References}

Barr, J. G. and Mahood, Rebecca J. (1976). An assessment of inositol fermentation by Klebsielleae and its implication in screening media. Journal of Clinical Pathology, 29, 827-832.

Braun, H. (1938). Ueber das aerobe und anaerobe Wachstum der Bakterien unter der Einwirkung von Kaliumzyanid: Colibazillen, Bacillus lactis aerogenes, Typhus-, Dysenteriebazillen und Choleravibrionen. Schweizerische Zeitschrift für Pathologie und Bakteriologie, 1, 257-266.

Chadwick, P., Delisle, G. J., and Byer, M. (1974). Biochemical identification of hospital enterobacteria by replica agar plating. Canadian Journal of Microbiology, 20, 1653-1664.

Cowan, S. T. (1974). Cowan and Steel's Manual for the Identification of Medical Bacteria. 2nd edition. Cambridge University Press, London.

Davis, T. J. and Matsen, J. M. (1974). Prevalence and characteristics of Klebsiella species: relation to association with a hospital environment. Journal of Infectious Diseases, 130, 402-405.

Donovan, T. J. (1966). A Klebsiella screening medium. Medical Laboratory Technology, 23, 194-197.

Fay, E. D. and Barry, A. L. (1972). Rapid ornithine decarboxylase test for the identification of Enterobacteriaceae. Applied Microbiology, 23, 710-713.

Møller, V. (1954). Diagnostic use of the Braun KCN test within the Enterobacteriaceae. Acta Pathologica et Microbiologica Scandinavica, 34, 115-126.

Oberhofer, T. R. and Hajkowski, R. (1970). Evaluation of non-lactose-fermenting members of the Klebsiella-Enterobacter-Serratia division: I. Biochemical characteristics. American Journal of Clinical Pathology, 54, 720-725.

Rosner, R. F. (1970). Identification of enteric bacilli directly from primary isolation media using reagent-impregnated paper strips. American Journal of Clinical Pathology, 54, 587-595.

Wasilauskus, B. L. (1971). Preliminary observations on the rapid differentiation of the Klebsiella-EnterobacterSerratia group on bile-esculin-agar. Applied Microbiology, 21, 162-163. 